\title{
Исследование помехозащищённости беспоискового спектрального корреляционно-интерферометрического радиопеленгатора для широкополосных сигналов
}

\begin{abstract}
На сегодняшний день в системах радиомониторинга пеленгование радиоэлектронных средств необходимо осуществлять в условиях сложной электромагнитной обстановки, большой априорной неопределенности относительно параметров радиоизлучений, а также в условиях реального времени. Перспективным направлением для указанных условий является использование беспоисковых моноимпульсных широкополосных корреляционно-интерферометрических радиопеленгаторов с применением цифровой обработки спектров принятой смеси радиоизлучений.

Проведен анализ помехозащищённости беспоискового спектрального корреляционноинтерферометрического радиопеленгатора, осуществляющего прямую оценку времени задержки полезного сигнала и соответствующего направления на источник радиоизлучения за один иикл корреляционной обработки. Проведен анализ основных вариантов помех $u$ электромагнитной обстановки, получены соответствующие аналитические оценки помехозащищённости пеленгования. Определены наиболее опасные варианть электромагнитной обстановки, при которых присутствует эффект «пространственной блокировки». B результате моделирования получено семейство зависимостей среднего квадратического отклонения оченки пеленга от отношения сигнал-помеха для разных типов электромагнитной обстановки. Результаты моделирования хорошо согласовываются с выражениями, полученными в результате теоретического анализа. Данные исследования являются продолжением работы по разработке беспоисковых спектральных методов
\end{abstract} корреляиионно-интерферометрического пеленгования.

Ключевые слова: анализ помехозащчищённости; беспоисковый цчифровой метод корреляционно-интерферометрического пеленгования; оценка дисперсии погрешности пеленгования.

Актуальность темы. На сегодняшний день в системах радиомониторинга пеленгование радиоэлектронных средств необходимо осуществлять в условиях сложной электромагнитной обстановки (ЭМО), большой априорной неопределенности относительно параметров радиоизлучений, а также в условиях реального времени. Перспективным направлением для указанных условий является использование беспоисковых моноимпульсных широкополосных корреляционно-интерферометрических радиопеленгаторов с применением цифровой обработки спектров принятой смеси радиоизлучений.

Анализ последних исследований и публикаций, на которые опираются авторы. Актуальной проблемой является радиомониторинг источников широкополосных радиоизлучений, в частности сверхширокополосных или с псевдослучайной перестройкой радиочастоты. В сложной ЭМО актуальной задачей является анализ помехозащищённости аппаратуры радиопеленгования. Следовательно, исследование помехозащищённости беспоисковых спектральных корреляционно-интерферометрических радиопеленгаторов для ЭМО разной сложности также является актуальной задачей.

В [4] разработан беспоисковый цифровой метод спектрального корреляционноинтерферометрического радиопеленгования с двойной корреляционной обработкой радиоизлучений с расширенным спектром, что даёт максимально правдоподобную оценку времени задержки полезного сигнала прямым аналитическим методом за один цикл корреляционной обработки. Это позволяет осуществлять корреляционно-интерферометрическую пеленгацию источников радиоизлучений с расширенным спектром и широкополосных радиоизлучений в условиях реального масштаба времени и использовать произвольную антенную базу. Однако исследование возможности использования такого метода в условиях сложной ЭМО не выполнено.

В [5-7] проведено исследование спектрально-энергетического метода разделения и выявления спектров сигналов для условий простой ЭМО, когда спектры сигналов разных источников радиоизлучений (ИРИ) в пределах полосы приёма не перекрываются и не перемежёвываются, с использованием классического критерия Рэлея или параметрических критериев. Однако исследование помехозащищённости пеленгования для ЭМО разной сложности не выполнено. 
В [8-10] проведено исследование пространственно-энергетического метода разделения, выявления и оценки параметров сигналов в принятой аддитивной смеси для условий сложной ЭМО, когда одновременно принимаются сигналы разных ИРИ, спектры которых перемежёвываются. Однако исследование помехозащищённости беспоискового спектрального корреляционноинтерферометрического радиопеленгатора с двойной корреляционной обработкой радиоизлучений, использующего двухэлементную антенную решётку, для указанных условий не выполнено.

В [11] для условий сложной ЭМО исследована помехоустойчивость беспоискового цифрового спектрального дисперсионно-корреляционного радиопеленгатора для большой антенной базы. Получена векторная оценка максимальной погрешности пеленгования данного метода. Однако особенности помехозащищённости других беспоисковых корреляционно-интерферометрических методов пеленгования не исследованы.

В [12] исследованы статистические характеристики и помехозащищённость классического многоитерационного корреляционно-интерферометрического радиопеленгатора. Однако особенности беспоискового спектрального корреляционно-интерферометрического пеленгования не исследованы.

Таким образом, для использования в автоматизованных системах радиомониторинга разработка и исследование помехозащищённости беспоисковых спектральных корреляционно-интерферометрических радиопеленгаторов является актуальной задачей.

Нерешённой ранее частью общей проблемы исследования помехозащищённости аппаратуры радиопеленгования является исследование помехозащищённости беспоискового спектрального корреляционно-интерферометрического радиопеленгатора с двойной корреляционной обработкой радиоизлучений с расширенным спектром, использующего два пеленгационных радиоканала.

Целью статьи является исследование помехозащищённости беспоискового спектрального корреляционно-интерферометрического радиопеленгатора с двойной корреляционной обработкой радиоизлучений с расширенным спектром.

Изложение основного материала. Выполним исследование помехозащищённости беспоискового спектрального корреляционно-интерферометрического радиопеленгатора с двойной корреляционной обработкой радиоизлучений, использующего два пеленгационных радиоканала.

Пусть осуществляется приём аддитивной смеси $U(t)$ излучений пеленгуемого полезного случайного широкополосного сигнала $S_{m}(t)$ и помехи $S_{z}(t)$ одновременно одной парой идентичных пеленгационных каналов радиопеленгатора, антенны которых разнесены в пространстве на расстояние антенной базы $d$ и имеют независимые аддитивные собственные шумы $n_{1}(t)$ и $n_{2}(t)$ соответственно.

Пусть $S_{1 m}(t)$ - реализация полезного сигнала $S_{m}(t)$, принятая в аддитивной смеси $U_{1}(t)$ со статистически независимыми реализациями помехи $S_{1 z}(t)$ и белого гауссовского шума $n_{1}(t)$ на конечном временном интервале $t \in\left[0, T_{a}\right]$ процесса анализа первым каналом, а $S_{2 m}(t)-$ реализация полезного сигнала $S_{m}(t)$, принятая в аддитивной смеси $U_{2}(t)$ со статистически независимыми реализациями помехи $S_{2 z}(t)$ и белого гауссовского шума $n_{2}(t)$ на конечном временном интервале $t \in\left[0, T_{a}\right]$ процесса анализа вторым каналом радиопеленгатора.

Приём аддитивных смесей $U_{1}(t)$ и $U_{2}(t)$ осуществляется первым и вторым радиоканалами соответственно в пределах полосы $\left[\omega_{L}, \omega_{H}\right]$ частот, равной их полосе пропускания или полосе одновременного анализа $[11,13]$. Источники радиоизлучений полезного сигнала $S_{m}(t)$ и помехи $S_{z}(t)$ разнесены в пространстве и соответствующие направления на них $\theta_{m}$ и $\theta_{z}$ относительно антенной базы $d$ радиопеленгатора являются независимыми случайными величинами с равномерным распределением плотности вероятности в заданном секторе $\theta=360^{\circ}$. Исходные условия целесообразно представить следующим образом:

$$
\begin{aligned}
& U_{1}(t)=S_{1 m}(t)+S_{1 z}(t)+n_{1}(t) \\
& U_{2}(t)=S_{1 m}\left(t-\tau_{S}\right)+S_{1 z}\left(t-\tau_{z}\right)+n_{2}(t),
\end{aligned}
$$

где $\tau_{S}, \tau_{z}-$ априори неизвестные величины относительного времени задержки приёма пеленгационными каналами полезного сигнала и помехи соответственно (далее - задержка полезного сигнала и помехи соответственно), являющиеся случайными величинами с равномерным распределением плотности вероятности на интервале $\left[-\tau_{\max } ; \tau_{\max }\right]$;

$\tau_{\max }=d / c$ - максимальное значение относительного времени задержки приёма радиоизлучений пеленгационными каналами, формируемой в случае прихода радиоизлучений с направления ноль градусов, отсчитанного от линии антенной базы. 
Будем считать, что полезный сигнал $S_{m}(t)$ и помеха $S_{z}(t)$ являются шумоподобными, имеют равномерный закон распределения их спектральных плотностей $S_{m}^{2}(\omega)$ и $S_{z}^{2}(\omega)$ мощности соответственно и антенная база $d$ больше их длины волн, то есть $d>\lambda_{m}, d>\lambda_{z}$, где $\lambda_{m}-$ длина волны полезного сигнала, $\lambda_{z}$ - длина волны помехи.

Для указанных условий (1) беспоисковый спектральный корреляционно-интерферометрический метод пеленгования с двойной корреляционной обработкой реализуется двойной цифровой корреляционной обработкой комплексных спектров $U_{1}\left(\mathrm{j} \omega_{I F . k}\right)$ и $U_{2}\left(\mathrm{j} \omega_{I F . k}\right)$ принятых аддитивных смесей $U_{1}(t)$ и $U_{2}(t)$ соответственно на промежуточной частоте $\omega_{I F}$ радиоканалов.

На первом этапе корреляционной обработки в цифровой форме анализируется на промежуточной частоте $\omega_{I F}$ первый взаимный комплексный спектр $U_{V 1}\left(\mathrm{j} \omega_{I F . k}\right)=U_{1}^{*}\left(\mathrm{j} \omega_{I F . k}\right) \cdot U_{2}\left(\mathrm{j} \omega_{I F . k}\right)$ принятых аддитивных смесей $U_{1}(t)$ и $U_{2}(t)$ с использованием временного сдвига.

На втором этапе корреляционной обработки анализируется двукратно-взаимный комплексный спектр $U_{V 2}\left(\mathrm{j} \omega_{I F . k}\right)=\left(U_{1}^{*}\left(\mathrm{j} \omega_{I F . k}\right) \cdot U_{2}\left(\mathrm{j} \omega_{I F . k}\right)\right)^{*} \cdot\left(U_{1}^{*}\left(\mathrm{j}\left(\omega_{I F . k}+\Delta \omega\right)\right) \cdot U_{2}\left(\mathrm{j}\left(\omega_{I F . k}+\Delta \omega\right)\right)\right)$ принятых аддитивных смесей $U_{1}(t)$ и $U_{2}(t)$ с использованием частотного преобразовательного сдвига $\Delta \omega$.

В результате обеспечивается возможность определения прямой беспоисковой оценки $\hat{\tau}_{S}$ относительного времени задержки приёма пеленгационными каналами полезного сигнала (далее оценка $\hat{\tau}_{S}$ задержки полезного сигнала) согласно уравнения [4]:

$$
\hat{\tau}_{S}=(1 / \Delta \omega) \cdot \operatorname{arctg}\left[\frac{\sum_{k=k_{L}}^{k_{H}} A_{1}\left(\omega_{I F . k}\right) \cdot A_{2}\left(\omega_{I F . k}\right) \cdot A_{1}\left(\omega_{I F . k}+\Delta \omega\right) \cdot A_{2}\left(\omega_{I F . k}+\Delta \omega\right) \cdot \sin \left[\Delta \varphi_{\Delta . k}\right]}{\sum_{k=k_{L}}^{k_{H}} A_{1}\left(\omega_{I F . k}\right) \cdot A_{2}\left(\omega_{I F . k}\right) \cdot A_{1}\left(\omega_{I F . k}+\Delta \omega\right) \cdot A_{2}\left(\omega_{I F . k}+\Delta \omega\right) \cdot \cos \left[\Delta \varphi_{\Delta . k}\right]}\right]+v \cdot \pi,
$$

где $k_{L}, k_{H}$ - номера частотных составляющих спектра сигнала, соответствующих нижней $\omega_{I F . L}$ и верхней $\omega_{I F . H}$ граничным частотам спектров сигналов на промежуточной частоте $\omega_{I F}$ радиоканалов соответственно;

$k \in[0 ;(N / 2)-1]$ - номера частотных спектральных составляющих входных аддитивных смесей радиоизлучений;

$N$ - количество отсчётов принятой аддитивной смеси радиоизлучений за время $T_{a}$ процесса анализа;

$A_{1}\left(\omega_{I F . k}\right), \quad A_{2}\left(\omega_{I F . k}\right)$ - амплитудные спектры принятых аддитивных смесей $U_{1}(t)$ и $U_{2}(t)$ на промежуточной частоте $\omega_{I F}$ в первом и втором радиоканалах соответственно;

$A_{1}\left(\omega_{I F . k}+\Delta \omega\right), A_{2}\left(\omega_{I F . k}+\Delta \omega\right)$ - амплитудные спектры принятых аддитивных смесей $U_{1}(t)$ и $U_{2}(t)$ на промежуточной частоте $\omega_{I F}$ после их частотного сдвига в первом и втором радиоканалах соответственно;

$\Delta \varphi_{\Delta . k}=\Delta \varphi\left(\omega_{I F . k}+\Delta \omega\right)-\Delta \varphi\left(\omega_{I F . k}\right)-$ двойной разностный фазовый сдвиг;

$\Delta \omega$ - значение частотного преобразовательного сдвига первого произведения спектров, кратное частотному шагу спектрального анализа;

$v-$ коэффициент коррекции неоднозначности для функции $\operatorname{arctg}():. v=0$ при $\cos (\Delta \varphi)>0 ; v=-1$ при $\cos (\Delta \varphi)<0$.

С учётом полученной оценки $\hat{\tau}_{S}$, формула (2), задержки полезного сигнала, дисперсия $\sigma_{\theta}^{2}$ погрешности оценки пеленга определяется следующим образом [13]:

$$
\sigma_{\theta}^{2}=c^{2} \cdot \sigma_{\tau}^{2} / d^{2} \cdot \cos ^{2} \theta,
$$

где $\sigma_{\tau}^{2}$ - дисперсия погрешности оценки задержки полезного сигнала.

Анализ уравнения (3) показывает, что принятые аддитивная помеха $S_{z}(t)$ и собственные шумы $n_{1}(t)$ и $n_{2}(t)$ пеленгационных каналов влияют непосредственно лишь на одну составляющую дисперсии $\sigma_{\theta}^{2}$ погрешности пеленгования, а именно на дисперсию $\sigma_{\tau}^{2}$ погрешности оценки задержки полезного сигнала. Поэтому этот параметр целесообразно использовать в качестве показателя помехозащищённости пеленгатора, учитывая его первичность и универсальность [11, 13]. 
Учитывая, что собственные шумы $n_{1}(t)$ и $n_{2}(t)$ пеленгационных каналов и помеха $S_{z}(t)$ являются статистически независимыми, дисперсия $\sigma_{\tau}^{2}$ погрешности оценки задержки полезного сигнала будет определяться двумя составляющими: шумовой и помеховой $[10,12,13]$ :

$$
\sigma_{\tau}^{2}=\sigma_{\tau n}^{2}+\sigma_{\tau z}^{2}
$$

где $\sigma_{\tau n}^{2}$ - шумовая составляющая дисперсии погрешности оценки задержки полезного сигнала;

$\sigma_{\tau z}^{2}-$ помеховая составляющая дисперсии погрешности оценки задержки полезного сигнала.

В свою очередь, шумовая составляющая $\sigma_{\tau n}^{2}$ дисперсии погрешности оценки задержки полезного сигнала определяется согласно уравнению [7, 12]:

$$
\sigma_{\tau n}^{2}=2 \pi / \Delta \omega^{2} \cdot T_{a} \cdot \mu \cdot \Delta \omega_{S},
$$

где $\mu$ - отношение сигнал/шум на входе пеленгационных каналов в принятых аддитивных смесях $U_{1}(t)$ i $U_{2}(t)$

$\Delta \omega_{S}$ - ширина спектра полезного сигнала.

Анализ уравнений (4) и (5) показывает, что шумовая составляющая $\sigma_{\tau n}^{2}$ дисперсии погрешности оценки задержки полезного сигнала определяется тремя основными факторами: отношением $\mu$ сигнал-шум, временем $T_{a}$ процесса анализа и шириной $\Delta \omega_{S}$ спектра полезного сигнала. При этом влияние действия собственных шумов может компенсироваться увеличением времени $T_{a}$ процесса анализа.

Для исследования помеховой составляющей $\sigma_{\tau z}^{2}$ дисперсии погрешности оценки задержки полезного сигнала целесообразно выделить следующие основные варианты ЭМО осуществления пеленгования: первый - спектр $S_{m}\left(\right.$ ј $\left.\omega_{I F . k}\right)$ полезного сигнала и спектр $S_{z}\left(\mathrm{j} \omega_{I F . k}\right)$ помехи не перекрываются; второй спектр $S_{m}\left(\mathrm{j} \omega_{I F . k}\right)$ полезного сигнала и спектр $S_{z}\left(\right.$ j $\left.\omega_{I F . k}\right)$ помехи перекрываются полностью; третий спектр $S_{m}\left(\mathrm{j} \omega_{I F . k}\right)$ полезного сигнала и спектр $S_{z}\left(\mathrm{j} \omega_{I F . k}\right)$ помехи перекрываются частично.

Для каждого из выделенных вариантов ЭМО целесообразно рассмотреть следующие типы помех: первый - узкополосная помеха, ширина $\Delta \omega_{z}$ спектра которой меньше ширины $\Delta \omega_{S}$ спектра полезного сигнала, то есть $\Delta \omega_{z}<\Delta \omega_{S}$; второй - широкополосная помеха, когда ширина $\Delta \omega_{z}$ её спектра больше ширины $\Delta \omega_{S}$ спектра полезного сигнала, то есть $\Delta \omega_{z}>\Delta \omega_{S}$; третий - равнополосная помеха, ширина спектра которой равна ширине спектра полезного сигнала, то есть $\Delta \omega_{z}=\Delta \omega_{S}$.

Первый тип помехи соответствует действию основного излучения узкополосных станционных помех или их паразитных составляющих на гармониках и субгармониках либо интермодуляционных $[1,8]$.

Второй тип помехи соответствует действию основного излучения широкополосных станционных помех современных средств связи и систем передачи информации, например типа UWB, с ортогональным частотным разделением каналов с мультиплексированием (OFDM), ППРЧ, использующих расширение спектра, или действию промышленных помех при осуществлении радиомониторинга в зоне их покрытия $[1,5]$.

Третий тип помехи является типичным для ЭМО населённых пунктов при многолучевом распространении излучений и формировании помех за счёт переотражений. Равнополосные коррелированные и некоррелированные с полезным сигналом помехи соответствуют, например, переотражённым собственным излучениям радиоэлектронных средств, пеленгуемым в текущий момент времени $[1,6,7]$.

Эпюры амплитудных спектров аддитивной смеси $U(t)$, соответствующие основным вариантам ЭМО, показаны на рисунках 1-3.

На рисунке 1 представлен первый вариант ЭМО осуществления пеленгования, при котором полоса $\left[\omega_{S . L} ; \omega_{S . H}\right]$ частот амплитудного спектра $S_{m}\left(\omega_{I F . k}\right)$ полезного сигнала и полоса $\left[\omega_{z . L} ; \omega_{z . H}\right]$ частот амплитудного спектра $S_{z}\left(\omega_{I F . k}\right)$ помехи не перекрываются, то есть $\omega_{S . H}<\omega_{z . L}$.

На рисунках 2 и 3 представлен второй вариант ЭМО осуществления пеленгования, при котором амплитудные спектры полезного сигнала и помехи перекрываются полностью. При этом на рисунке 2 представлена эпюра аддитивной смеси $U(t)$, включающей широкополосную помеху с шириной $\Delta \omega_{z}=\omega_{z . H}-\omega_{z . L}$ полосы частот, большей ширины $\Delta \omega_{S}=\omega_{S . H}-\omega_{S . L}$ полосы частот полезного сигнала. На рисунке 3 представлена эпюра аддитивной смеси $U(t)$, включающей узкополосную помеху с шириной $\Delta \omega_{z}$ полосы частот, меньшей ширины $\Delta \omega_{S}$ полосы частот полезного сигнала. 


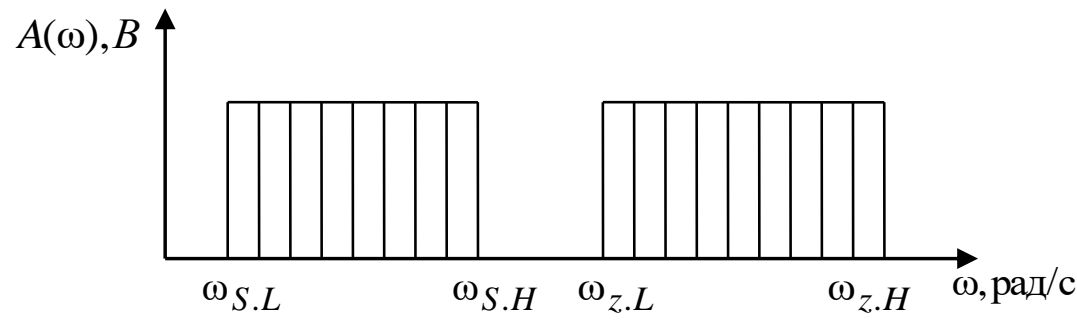

Рис. 1. Эпюры амплитудных спектров полезного сигнала и помехи, не перекрываюшихся по частоте

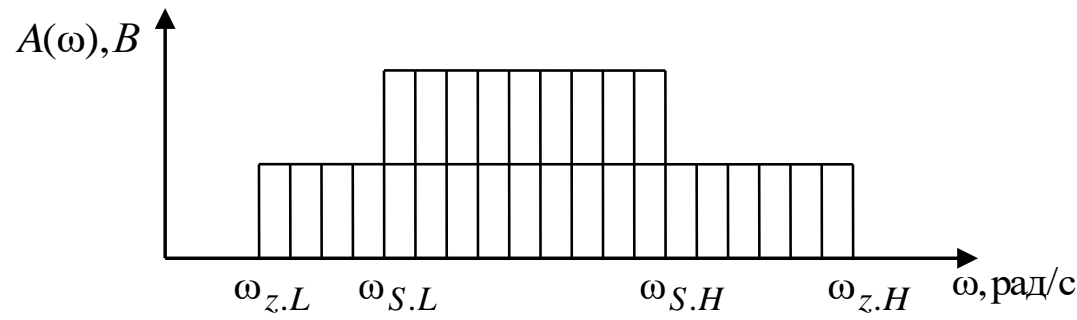

Рис. 2. Эпюры амплитудных спектров полезного сигнала и широкополосной помехи, перекрываюшихся по частоте полностью

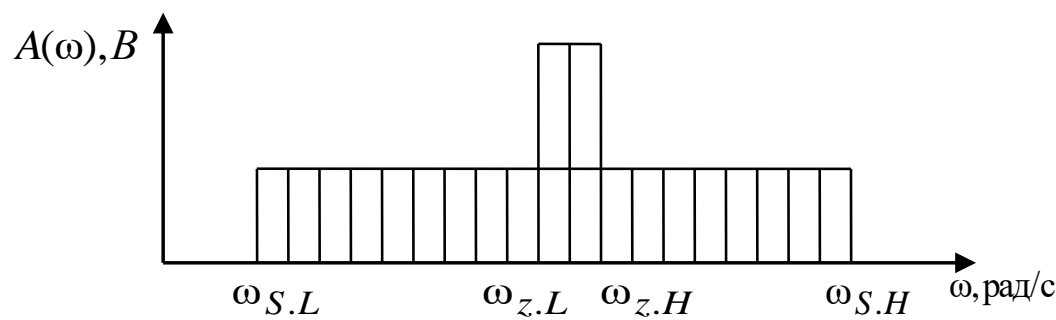

Рис. 3. Эпюры амплитудных спектров полезного сигнала и узкополосной помехи, перекрываюшихся по частоте полностью

Выполним исследование помеховой составляющей $\sigma_{\tau z}^{2}$ дисперсии погрешности оценки задержки полезного сигнала для первого варианта ЭМО, когда спектры полезного сигнала и помехи не перекрываются, являются смежными и имеют одинаковую ширину. Условия исследований представим следующим образом:

$$
\left\{\begin{array}{l}
\Delta \omega_{S}=\Delta \omega_{z} ; \\
\omega_{S . H}<\omega_{z . L} ; \\
\Delta \omega_{a}=\Delta \omega_{S}+\Delta \omega_{z} ; \\
\Delta \omega_{a} \leq \Delta \omega_{k},
\end{array}\right.
$$

где $\Delta \omega_{a}$ - ширина полосы частот одновременного анализа;

$\Delta \omega_{k}-$ ширина полосы пропускания пеленгационных каналов.

С учётом действия только помехи комплексные спектры $U_{1}\left(\mathrm{j} \omega_{I F . k}\right)$ и $U_{2}\left(\mathrm{j} \omega_{I F . k}\right)$, формирующиеся на основе быстрого преобразования Фурье на промежуточной частоте в первом и втором пеленгационных каналах соответственно, определяются следующим образом:

$$
\begin{aligned}
& U_{1}\left(\mathrm{j} \omega_{I F . k}\right)=S_{1 m}\left(\mathrm{j} \omega_{I F . k}\right)+S_{1 z}\left(\mathrm{j} \omega_{I F . k}\right)+n_{1}\left(\mathrm{j} \omega_{I F . k}\right) ; \\
& U_{2}\left(\mathrm{j} \omega_{I F . k}\right)=S_{2 m}\left(\mathrm{j} \omega_{I F . k}\right)+S_{2 z}\left(\mathrm{j} \omega_{I F . k}\right)+n_{2}\left(\mathrm{j} \omega_{I F . k}\right),
\end{aligned}
$$

где $S_{1 m}\left(\mathrm{j} \omega_{I F . k}\right), S_{2 m}\left(\mathrm{j} \omega_{I F . k}\right)$ - комплексные спектры полезных сигналов на промежуточной частоте первого и второго пеленгационных каналов соответственно;

$S_{1 z}\left(\mathrm{j} \omega_{I F . k}\right), S_{2 z}\left(\mathrm{j} \omega_{I F . k}\right)$ - комплексные спектры помехи в первом и втором пеленгационных каналах соответственно;

$n_{1}\left(\mathrm{j} \omega_{I F . k}\right), \quad n_{2}\left(\mathrm{j} \omega_{I F . k}\right) \quad-$ комплексные спектры собственных шумов в первом и втором пеленгационых каналах соответственно. 
После двойной корреляционной обработки комплексных спектров $U_{1}\left(\mathrm{j} \omega_{I F . k}\right) \quad$ и $U_{2}\left(\mathrm{j} \omega_{I F . k}\right)$ принятых аддитивных смесей $U_{1}(t)$ и $U_{2}(t)$ на промежуточной частоте оценка $\hat{\tau}_{S}$ задержки полезного сигнала для условий (6) определяется с учётом (2) и (7) следующим образом:

$$
\begin{aligned}
& \hat{\tau}_{S}=\left(1 / \Delta \omega_{a}\right) \times \\
& \times \operatorname{arctg}\left(\frac{\sum_{k=k_{L}}^{k_{H}} S_{1 m}\left(\omega_{I F . k}\right) \cdot S_{2 m}\left(\omega_{I F . k}\right) \cdot S_{1 z}\left(\omega_{I F . k}+\Delta \omega\right) \cdot S_{2 z}\left(\omega_{I F . k}+\Delta \omega\right) \cdot \sin \left(\Delta \varphi_{\Delta 1 . k}\right)}{\sum_{k=k_{L}}^{k_{H}} S_{1 m}\left(\omega_{I F . k}\right) \cdot S_{2 m}\left(\omega_{I F . k}\right) \cdot S_{1 z}\left(\omega_{I F . k}+\Delta \omega\right) \cdot S_{2 z}\left(\omega_{I F . k}+\Delta \omega\right) \cdot \cos \left(\Delta \varphi_{\Delta 1 . k}\right)}\right)+ \\
& +v \cdot \pi=\tau_{z}-\tau_{S},
\end{aligned}
$$

где $\Delta \varphi_{\Delta 1 . k}=\Delta \varphi_{z}\left(\omega_{I F . k}+\Delta \omega\right)-\Delta \varphi_{S}\left(\omega_{I F . k}\right)$

$\Delta \varphi_{S}\left(\omega_{I F . k}\right)=\varphi_{2 S}\left(\omega_{I F, k}\right)-\varphi_{1 S}\left(\omega_{I F . k}\right)-$ аргумент взаимного спектра полезного сигнала;

$\Delta \varphi_{z}\left(\omega_{I F . k}+\Delta \omega\right)=\varphi_{2 z}\left(\omega_{I F . k}+\Delta \omega\right)-\varphi_{1 z}\left(\omega_{I F . k}+\Delta \omega\right)$ - аргумент взаимного спектра помехи;

$\varphi_{1 S}\left(\omega_{I F . k}\right), \varphi_{2 S}\left(\omega_{I F . k}\right)$ - аргументы комплексных спектров $S_{1 m}\left(\mathrm{j} \omega_{I F . k}\right), \quad S_{2 m}\left(\mathrm{j} \omega_{I F . k}\right)$ полезного сигнала на промежуточной частоте первого и второго пеленгационных каналов соответственно;

$\varphi_{1 z}\left(\omega_{I F . k}+\Delta \omega\right), \quad \varphi_{2 z}\left(\omega_{I F . k}+\Delta \omega\right) \quad-\quad$ аргументы $\quad$ комплексных $\quad$ спектров $\quad S_{1 z}\left(\mathrm{j}\left(\omega_{I F . k}+\Delta \omega\right)\right)$, $S_{2 z}\left(\mathrm{j}\left(\omega_{I F . k}+\Delta \omega\right)\right)$ помехи на промежуточной частоте первого и второго пеленгационных каналов соответственно;

$\Delta \omega=\Delta \omega_{a} / 2-$ частотный сдвиг при повторной корреляционной обработке.

Анализ уравнения (8) показывает, что за счёт двойной корреляционной обработки комплексных спектров $U_{1}\left(\mathrm{j} \omega_{I F, k}\right) \quad$ и $U_{2}\left(\mathrm{j} \omega_{I F, k}\right)$ аргументы $\Delta \varphi_{\Delta 1 . k}$ сформированного двукратно-взаимного комплексного спектра $U_{V 2}\left(\mathrm{j} \omega_{I F . k}\right)$ равняются разности аргументов взаимных спектров полезного сигнала и помехи. Это, в свою очередь, обусловливает появление аномально большой погрешности оценки $\hat{\tau}_{S}$ задержки полезного сигнала. В результате данное влияние помехи целесообразно определить как «пространственную блокировку» полезного сигнала, при которой возникает потеря пространственной информации о полезном сигнале и соответствующей задержке $\tau_{S}$ полезного сигнала и, как следствие, эквивалентное уменьшение мощности полезного сигнала независимо от соотношения мощности $P_{S}$ полезного сигнала и мощности $P_{z}$ помехи, в том числе при условии $P_{S} / P_{z}>1$.

Учитывая равномерный закон распределения плотности вероятности аргумента $\Delta \varphi_{z}\left(\omega_{I F . k}+\Delta \omega\right)$ взаимного спектра помехи, помеховая составляющая $\sigma_{\tau z}^{2}$ дисперсии погрешности оценки задержки полезного сигнала с учётом (8) составляет [10]:

$$
\sigma_{\tau z}^{2}=\tau_{\max }^{2} / 3 .
$$

Эффект «пространственной блокировки» можно эффективно устранить с помощью предварительной селекции, например, амплитудной или пространственной, спектральных составляющих помехи $U_{z}\left(\mathrm{j} \omega_{I F . k}\right)$ из комплексных спектров $U_{1}\left(\mathrm{j} \omega_{I F . k}\right)$ и $U_{2}\left(\mathrm{j} \omega_{I F . k}\right)$ принятых аддитивных смесей $U_{1}(t)$ и $U_{2}(t)$ на промежуточной частоте $[5,8]$.

Рассмотрим первый вариант ЭМО при действии узкополосной помехи, которая не перекрывается по частоте с полезным сигналом. Эти условия определим следующим образом:

$$
\left\{\begin{array}{l}
\Delta \omega_{S}>\Delta \omega_{z} ; \\
\Delta \omega_{a}=\Delta \omega_{S}+\Delta \omega_{z} ; \\
S_{z}\left(\mathrm{j} \omega_{k}\right) \notin\left[\omega_{S . L} ; \omega_{S . H}\right] .
\end{array}\right.
$$

В случае (10) эффект «пространственной блокировки» спектральных составляющих взаимного спектра полезного сигнала будет только частичным. В результате информация о направлении $\theta_{S}$ прихода полезного сигнала будет потеряна только для спектральных составляющих взаимного комплексного спектра $S_{1 m}\left(\omega_{I F . k}\right) \cdot S_{1 m}\left(\omega_{I F . k}+\Delta \omega\right) \cdot \exp \left(\mathrm{j} \Delta \varphi_{S k}\right)$ полезного сигнала, которые совпадают по частоте с преобразованным взаимным комплексным спектром помехи $S_{1 z}\left(\mathrm{j} \omega_{I F . k}\right) \cdot S_{1 z}\left(\mathrm{j}\left(\omega_{I F . k}+\Delta \omega\right)\right)$, то есть в пределах полосы $\Delta \omega_{z}$ частот. В пределах полосы $\left(\Delta \omega_{S}-\Delta \omega_{z}\right)$ частот эффект «пространственной блокировки» будет отсутствовать.

Таким образом, в результате двойной корреляционной обработки комплексных спектров $U_{1}\left(\mathrm{j} \omega_{I F . k}\right)$ и $U_{2}\left(\mathrm{j} \omega_{I F . k}\right)$ для условий (10) будут сформированы два суммарных комплексных вектора: сигнальный и 
помеховый, соотношение которых будет определять помеховую составляющую $\sigma_{\tau z}^{2}$ дисперсии погрешности оценки задержки полезного сигнала.

Оценка $\hat{\tau}_{S}$ задержки полезного сигнала в этом случае определяется следующим образом:

$$
\hat{\tau}_{S}=\left(1 / \Delta \omega_{a}\right) \cdot \operatorname{arctg}\left(\frac{\sum_{k=k_{L}}^{k_{H}} S_{z \Sigma} \cdot \sin \left(\Delta \varphi_{z \Sigma}\right)+S_{m \Sigma} \cdot \sin \left(\Delta \varphi_{m \Sigma}\right)}{\sum_{k=k_{L}}^{k_{H}} S_{z \Sigma} \cdot \sin \left(\Delta \varphi_{z \Sigma}\right)+S_{m \Sigma} \cdot \sin \left(\Delta \varphi_{m \Sigma}\right)}\right)+v \cdot \pi,
$$

где $\dot{S}_{z \Sigma}=S_{z \Sigma} \cdot \exp \left(\mathrm{j} \Delta \varphi_{z \Sigma}\right)$ - суммарный помеховый комплексный вектор;

$\dot{S}_{m \Sigma}=S_{m \Sigma} \cdot \exp \left(\mathrm{j} \Delta \varphi_{m \Sigma}\right)-$ суммарный сигнальный комплексный вектор.

Суммарный помеховый комплексный вектор $S_{z \Sigma} \cdot \exp \left(\mathrm{j} \Delta \varphi_{z \Sigma}\right)$ является случайным с нормальным распределением плотности вероятности, поэтому с учётом (11) помеховая составляющая $\sigma_{\tau z}^{2}$ дисперсии погрешности оценки задержки полезного сигнала равняется [7, 9]:

$$
\sigma_{\tau z}^{2}=\left(\frac{2 P_{z}}{P_{S}\left(1-\Delta \omega_{z} / \Delta \omega_{S}\right)}\right)^{2} .
$$

Анализ уравнения (12) показывает, что помеховая составляющая $\sigma_{\tau z}^{2}$ дисперсии погрешности оценки задержки полезного сигнала определяется отношением сигнал-помеха $\left(P_{S} / P_{z}\right)$ по мощности и отношением ширины спектра помехи и сигнала $\left(\Delta \omega_{z} / \Delta \omega_{S}\right)$. При этом с увеличением ширины $\Delta \omega_{z}$ спектра помехи помеховая составляющая $\sigma_{\tau z}^{2}$ дисперсии погрешности оценки задержки полезного сигнала существенно, по квадратичной зависимости, растет. Для данного варианта ЭМО уменьшение помеховой составляющей $\sigma_{\tau z}^{2}$ дисперсии погрешности оценки задержки полезного сигнала за счёт увеличения времени $T_{a}$ процесса анализа невозможно.

Рассмотрим первый вариант ЭМО при действии широкополосной помехи, когда $\Delta \omega_{z}>\Delta \omega_{S}$, и при отсутствии перекрытия её спектра по частоте со спектром полезного сигнала. Эффект «пространственной блокировки» возникает для всех спектральных составляющих полезного сигнала $S_{m}\left(\mathrm{j} \omega_{I F . k}\right)$ в пределах полосы $\Delta \omega_{S}$. В результате этого аномально увеличивается помеховая составляющая $\sigma_{\tau z}^{2}$ дисперсии погрешности оценки задержки полезного сигнала аналогично уравнению (9).

Таким образом, приём помехи, спектр которой не перекрывается по частоте со спектром полезного сигнала, существенно ухудшает точность пеленгования, которую нельзя улучшить увеличением времени $T_{a}$ процесса анализа. Эффективным методом улучшения помехозащищённости исследуемого метода пеленгования для данного варианта ЭМО является использование предварительной селекции, например, амплитудной или пространственной, спектра помехи с последующим уточнением границ полосы $\Delta \omega_{a}$ одновременного анализа.

Выполним исследование помеховой составляющей $\sigma_{\tau z}^{2}$ дисперсии погрешности оценки задержки полезного сигнала для второго варианта ЭМО, когда спектры полезного сигнала и помехи полностью перекрываются, имеют одинаковую ширину полосы частот, равную ширине полосы $\Delta \omega_{a}=\left[\omega_{a . L} ; \omega_{a . H}\right]$ одновременного анализа, но помеха является некоррелированной с полезным сигналом, то есть:

$$
\left\{\begin{array}{l}
\Delta \omega_{S}=\Delta \omega_{z}=\Delta \omega_{a} ; \\
{\left[\omega_{z . L} ; \omega_{z . H}\right] \in\left[\omega_{S . L} ; \omega_{S . H}\right] \in\left[\omega_{a . L} ; \omega_{a . H}\right] .}
\end{array}\right.
$$

Для заданных условий (13) на первом и втором этапах корреляционной обработки определяются соответственно первый $U_{1 V}\left(\mathrm{j} \omega_{I F . k}\right)$ и второй $U_{2 V}\left(\mathrm{j} \omega_{I F . k}\right)$ взаимные спектры полученных комплексных спектров $U_{1}\left(\mathrm{j} \omega_{I F . k}\right)$ и $U_{2}\left(\mathrm{j} \omega_{I F . k}\right)$.

Полученный в результате двойной корреляционной обработки двукратно-взаимный комплексный спектр $U_{2 V}\left(\mathrm{j} \omega_{I F . k}\right)$ при отношении сигнал / помеха $P_{S} / P_{z}>1$ содержит следующие четыре значимые составляющие: 


$$
\begin{aligned}
& U_{2 V}\left(\mathrm{j} \omega_{I F . k}\right)=\left[\left[S_{1 m}\left(\mathrm{j} \omega_{I F . k}\right)+S_{1 z}\left(\mathrm{j} \omega_{I F . k}\right)\right]^{*} \cdot\left[S_{2 m}\left(\mathrm{j} \omega_{I F . k}\right)+S_{2 z}\left(\mathrm{j} \omega_{I F . k}\right)\right]\right]^{*} \times \\
& \times\left[\left[S_{1 m}\left(\mathrm{j}\left(\omega_{I F . k}+\Delta \omega\right)\right)+S_{1 z}\left(\mathrm{j}\left(\omega_{I F . k}+\Delta \omega\right)\right)\right]^{*} \cdot\left[S_{2 m}\left(\mathrm{j}\left(\omega_{I F . k}+\Delta \omega\right)\right)+S_{2 z}\left(\mathrm{j}\left(\omega_{I F . k}+\Delta \omega\right)\right)\right]\right]= \\
& =\left[S_{1 m}^{*}\left(\mathrm{j} \omega_{I F . k}\right) \cdot S_{2 m}\left(\mathrm{j} \omega_{I F . k}\right)\right]^{*} \cdot S_{1 m}^{*}\left(\mathrm{j}\left(\omega_{I F . k}+\Delta \omega\right)\right) \cdot S_{2 m}\left(\mathrm{j}\left(\omega_{I F . k}+\Delta \omega\right)\right)+ \\
& +\left[S_{1 m}^{*}\left(\mathrm{j} \omega_{I F . k}\right) \cdot S_{2 m}\left(\mathrm{j} \omega_{I F . k}\right)\right]^{*} \cdot S_{1 z}^{*}\left(\mathrm{j}\left(\omega_{I F . k}+\Delta \omega\right)\right) \cdot S_{2 z}\left(\mathrm{j}\left(\omega_{I F . k}+\Delta \omega\right)\right)+ \\
& +\left[S_{1 z}^{*}\left(\mathrm{j} \omega_{I F . k}\right) \cdot S_{2 z}\left(\mathrm{j} \omega_{I F . k}\right)\right]^{*} \cdot S^{*}{ }_{1 m}\left(\mathrm{j}\left(\omega_{I F . k}+\Delta \omega\right)\right) \cdot S_{2 m}\left(\mathrm{j}\left(\omega_{I F . k}+\Delta \omega\right)\right)+ \\
& +\left[S_{1 z}^{*}\left(\mathrm{j} \omega_{I F . k}\right) \cdot S_{2 z}\left(\mathrm{j} \omega_{I F . k}\right)\right]^{*} \cdot S^{*}{ }_{1 z}\left(\mathrm{j}\left(\omega_{I F . k}+\Delta \omega\right)\right) \cdot S_{2 z}\left(\mathrm{j}\left(\omega_{I F . k}+\Delta \omega\right)\right)
\end{aligned}
$$

При увеличении мощности $P_{z}$ помехи в аддитивной смеси $U(t)$ количество существенных помеховых составляющих после двойной корреляционной обработки будет расти и может достигать пятнадцати. Однако наибольший интерес представляет случай, когда мощность $P_{S}$ полезного сигнала существенно больше мощности $P_{z}$ помехи в принятых аддитивных смесях $U_{1}(t)$ и $U_{2}(t)$, то есть $P_{S} / P_{z}>>1$, что соответствует случаю принятых эксплуатационных условий по точности пеленгования.

Анализ уравнения (14) показывает, что за счёт некоррелированности составляющих спектров полезного сигнала и помехи, а также разных частотных составляющих спектра помехи, после двойной корреляционной обработки значительное количество составляющих двукратно-взаимного комплексного спектра $U_{2 V}\left(\mathrm{j} \omega_{I F . k}\right)$ может не учитываться, а итоговая помеховая составляющая $U_{2 V z}\left(\mathrm{j} \omega_{I F . k}\right)$ при условии $\left(P_{S}>P_{z}\right)$ равняется:

$$
\begin{aligned}
& U_{2 V z}\left(\mathrm{j} \omega_{I F . k}\right)=\left[S^{*}{ }_{1 m}\left(\mathrm{j} \omega_{I F . k}\right) \cdot S_{2 m}\left(\mathrm{j} \omega_{I F . k}\right)\right]^{*} \cdot S^{*}{ }_{1 z}\left(\mathrm{j}\left(\omega_{I F . k}+\Delta \omega\right)\right) \cdot S_{2 z}\left(\mathrm{j}\left(\omega_{I F . k}+\Delta \omega\right)\right)+ \\
& +\left[S^{*}{ }_{1 z}\left(\mathrm{j} \omega_{I F . k}\right) \cdot S_{2 z}\left(\mathrm{j} \omega_{I F . k}\right)\right]^{*} \cdot S^{*}{ }_{1 m}\left(\mathrm{j}\left(\omega_{I F . k}+\Delta \omega\right)\right) \cdot S_{2 m}\left(\mathrm{j}\left(\omega_{I F . k}+\Delta \omega\right)\right)
\end{aligned}
$$

С учётом (15) оценка $\hat{\tau}_{S} \quad$ задержки полезного сигнала при $S_{1 m}\left(\omega_{I F . k}\right)=S_{2 m}\left(\omega_{I F . k}\right) \quad$ и $S_{1 z}\left(\omega_{I F . k}+\Delta \omega\right)=S_{2 z}\left(\omega_{I F . k}+\Delta \omega\right)$ в этом случае определяется следующим образом:

$$
\hat{\tau}_{S}=\left(1 / \Delta \omega_{a}\right) \cdot \operatorname{arctg}\left(\begin{array}{l}
\sum_{k=k_{L}}^{k_{H}} S_{1 m}^{2}\left(\omega_{I F . k}\right) \cdot S_{1 m}^{2}\left(\omega_{I F . k}+\Delta \omega\right) \cdot \sin \left(\Delta \varphi_{\Delta 2 . k}\right)+ \\
+\sum_{k=k_{L}}^{k_{H}} S_{1 m}^{2}\left(\omega_{I F . k}\right) \cdot S_{1 z}^{2}\left(\omega_{I F . k}+\Delta \omega\right) \cdot \sin \left(\Delta \varphi_{\Delta 3 . k}\right)+ \\
+\sum_{k=k_{L}}^{k_{H}} S_{1 z}^{2}\left(\omega_{I F . k}\right) \cdot S_{1 m}^{2}\left(\omega_{I F . k}+\Delta \omega\right) \cdot \sin \left(\Delta \varphi_{\Delta 4 . k}\right) \\
\sum_{k=k_{L}}^{k_{H}} S_{1 m}^{2}\left(\omega_{I F . k}\right) \cdot S_{1 m}^{2}\left(\omega_{I F . k}+\Delta \omega\right) \cdot \cos \left(\Delta \varphi_{\Delta 2 . k}\right)+ \\
+\sum_{k=k_{L}}^{k_{H}} S_{1 m}^{2}\left(\omega_{I F . k}\right) \cdot S_{1 z}^{2}\left(\omega_{I F . k}+\Delta \omega\right) \cdot \cos \left(\Delta \varphi_{\Delta 3 . k}\right)+ \\
+\sum_{k=k_{L}}^{k_{H}} S_{1 z}^{2}\left(\omega_{I F . k}\right) \cdot S_{1 m}^{2}\left(\omega_{I F . k}+\Delta \omega\right) \cdot \cos \left(\Delta \varphi_{\Delta 4 . k}\right)
\end{array}\right),
$$

где $\Delta \varphi_{\Delta 2 . k}=\Delta \varphi_{S}\left(\omega_{I F . k}+\Delta \omega\right)-\Delta \varphi_{S}\left(\omega_{I F . k}\right)$;

$\Delta \varphi_{\Delta 3 . k}=\Delta \varphi_{z}\left(\omega_{I F . k}+\Delta \omega\right)-\Delta \varphi_{S}\left(\omega_{I F . k}\right) ;$

$\Delta \varphi_{\Delta 4 . k}=\Delta \varphi_{S}\left(\omega_{I F . k}+\Delta \omega\right)-\Delta \varphi_{z}\left(\omega_{I F . k}\right)$.

Суммарное взаимодействие $\vec{U}_{\Sigma . H}$ сигнала и некоррелированной помехи в (16) целесообразно представить в векторной форме: $\vec{U}_{\Sigma . H}=\vec{S}_{m . H}+\vec{S}_{z . H}$ [11]. Длины векторов полезного сигнала $\left|\vec{S}_{m . H}\right|$ и помехи $\left|\vec{S}_{z . H}\right|$ при действии некоррелированной с полезным сигналом помехи будут равняться: 


$$
\begin{gathered}
\left|\vec{S}_{m . H}\right|=\left|\sum_{k=k_{L}}^{k_{H}} S_{1 m}^{2}\left(\omega_{I F, k}\right) \cdot S_{1 m}^{2}\left(\omega_{I F, k}+\Delta \omega\right) \cdot \exp \left[j \Delta \varphi_{\Delta 2 k}\right]\right| ; \\
\left|\vec{S}_{z . H}\right|=\left|\begin{array}{l}
\sum_{k=k_{L}}^{k_{H}} S_{1 m}^{2}\left(\omega_{I F . k}\right) \cdot S_{1 z}^{2}\left(\omega_{I F . k}+\Delta \omega\right) \cdot \exp \left(j \Delta \varphi_{\Delta 3 k}\right)+ \\
+\sum_{k=k_{L}}^{k_{H}} S_{1 z}^{2}\left(\omega_{I F . k}\right) \cdot S_{1 m}^{2}\left(\omega_{I F . k}+\Delta \omega\right) \exp \left(j \Delta \varphi_{\Delta 4 k}\right)
\end{array}\right| .
\end{gathered}
$$

Значение максимальной погрешности $\Delta \alpha$ оценки аргумента сигнала полезного вектора, то есть отклонение аргумента суммарного вектора $\vec{S}_{m . H}$ сигнала от аргумента суммарного вектора $\vec{U}_{\Sigma . H}$ под действием помехи при величине разностного угла $\alpha_{z}-\alpha_{m}= \pm 90^{\circ}$ аргументов соответственно помехового и сигнального суммарных векторов будет равняться [11]:

$$
\Delta \alpha=\operatorname{arctg}\left(\left|\vec{S}_{z . H}\right| /\left|\vec{S}_{m . H}\right|\right) \text {. }
$$

Также анализ уравнения (17) и (18) показывает, что помеховая составляющая $\vec{S}_{z . H}$ оценки задержки полезного сигнала является случайным нормальным вектором, плотность вероятности модуля которого распределена по закону Рэлея, а плотность вероятности аргумента - по равномерному закону $[9,10]$. В результате помеховая составляющая $\sigma_{\tau z}^{2}$ дисперсии погрешности оценки задержки полезного сигнала при действии некоррелированной помехи, спектр которой перекрывается по частоте со спектром полезного сигнала, определяется отношением сигнал / помеха $\left(P_{S} / P_{z}\right)$ и равняется:

$$
\sigma_{\tau z}^{2}=\left(2 P_{z} / P_{S}\right)^{2} .
$$

Сравнительный анализ оценок (5) и (19) показывает, что в отличие от действия собственных шумов, помехозащищённость исследуемого пеленгатора при приёме помехи, спектр которой перекрывается по частоте со спектром полезного сигнала, определяется двойным отношением сигнал/помеха и не улучшается увеличением времени $T_{a}$ процесса анализа.

Выполним анализ помехозащищённости для условий (13) при действии коррелированной с полезным сигналом помехи, что является типичным вариантом условий пеленгования при наличии многолучевого распространения радиоизлучений в условиях города $[1,2,6]$.

Пусть коэффициент $K_{v}$ ослабления на трассе распространения является случайной величиной с Рэлеевским законом распределения плотности вероятности. В этом случае состав двукратно-взаимного комплексного спектра $U_{V 2}\left(\mathrm{j} \omega_{I F, k}\right)$ существенно изменится по сравнению с (16) и в первом приближении будет определяться согласно уравнению:

$$
\begin{aligned}
& U_{V 2}\left(\mathrm{j} \omega_{I F, k}\right)=\left[\left[S_{1 m}\left(\mathrm{j} \omega_{k}\right)+S_{1 z}\left(\mathrm{j} \omega_{k}\right)\right]^{*} \cdot\left[S_{2 m}\left(\mathrm{j} \omega_{k}\right)+S_{2 z}\left(\mathrm{j} \omega_{k}\right)\right]\right]^{*} \times \\
& \times\left[\left[S_{1 m}\left(\mathrm{j}\left(\omega_{k}+\Delta \omega\right)\right)+S_{1 z}\left(\mathrm{j}\left(\omega_{k}+\Delta \omega\right)\right)\right]^{*} \cdot\left[S_{2 m}\left(\mathrm{j}\left(\omega_{k}+\Delta \omega\right)\right)+S_{2 z}\left(\mathrm{j}\left(\omega_{k}+\Delta \omega\right)\right)\right]\right]= \\
& =\left[S^{*}{ }_{1 m}\left(\mathrm{j} \omega_{k}\right) \cdot S_{2 m}\left(\mathrm{j} \omega_{k}\right)\right]^{*} \cdot S^{*}{ }_{1 m}\left(\mathrm{j}\left(\omega_{k}+\Delta \omega\right)\right) \cdot S_{2 m}\left(\mathrm{j}\left(\omega_{k}+\Delta \omega\right)\right)+ \\
& +\left[S^{*}{ }_{1 m}\left(\mathrm{j} \omega_{k}\right) \cdot S_{2 m}\left(\mathrm{j} \omega_{k}\right)\right]^{*} \cdot S^{*}{ }_{1 m}\left(\mathrm{j}\left(\omega_{k}+\Delta \omega\right)\right) \cdot S_{2 z}\left(\mathrm{j}\left(\omega_{k}+\Delta \omega\right)\right)+ \\
& +\left[S^{*}{ }_{1 m}\left(\mathrm{j} \omega_{k}\right) \cdot S_{2 m}\left(\mathrm{j} \omega_{k}\right)\right]^{*} \cdot S^{*}{ }_{1 z}\left(\mathrm{j}\left(\omega_{k}+\Delta \omega\right)\right) \cdot S_{2 m}\left(\mathrm{j}\left(\omega_{k}+\Delta \omega\right)\right)+ \\
& +\left[S^{*}{ }_{1 m}\left(\mathrm{j} \omega_{k}\right) \cdot S_{2 z}\left(\mathrm{j} \omega_{k}\right)\right]^{*} \cdot S_{1 m}^{*}\left(\mathrm{j}\left(\omega_{k}+\Delta \omega\right)\right) \cdot S_{2 m}\left(\mathrm{j}\left(\omega_{k}+\Delta \omega\right)\right)+ \\
& +\left[S^{*}{ }_{1 z}\left(\mathrm{j} \omega_{k}\right) \cdot S_{2 m}\left(\mathrm{j} \omega_{k}\right)\right]^{*} \cdot S^{*}{ }_{1 m}\left(\mathrm{j}\left(\omega_{k}+\Delta \omega\right)\right) \cdot S_{2 m}\left(\mathrm{j}\left(\omega_{k}+\Delta \omega\right)\right)
\end{aligned}
$$

Анализ уравнения (20) показывает, что помеховая составляющая $U_{V 2 z}$ двукратно-взаимного комплексного спектра содержит четыре основных составляющих, которые являются случайными комплексными векторами, аргументы которых некоррелированны.

В результате помеховая составляющая $\sigma_{\tau z}^{2}$ дисперсии погрешности оценки задержки полезного сигнала будет равняться:

$$
\sigma_{\tau z}^{2}=4 P_{z} / P_{S}=16 K_{v}^{2} .
$$

Анализ уравнения (21) показывает, что при беспоисковом пеленговании с двойной корреляционной обработкой осуществляется одновременное накопление энергии полезного сигнала и помехи. Поэтому погрешность пеленгования, обусловленная действием коррелированной помехи, также не может быть уменьшена путем увеличения времени $T_{a}$ процесса анализа в отличие от случая действия только 
собственных шумов. Сравнительный анализ выражений (19) и (20) показывает, что помехозащищённость при действии коррелированной помехи значительно хуже по сравнению с частотно разнесённой и некоррелированной помехой за счёт действия взаимных корреляционных составляющих сигнала и помехи. При этом погрешность пеленгования при действии коррелированной помехи может быть уменьшена за счёт использования предварительной селекции помехи, например, пространственной или поляризационной.

При действии узкополосной помехи при втором варианте ЭМО начальные условия анализа определяются согласно уравнениям:

$$
\left\{\begin{array}{l}
\Delta \omega_{a}=\Delta \omega_{S} ; \\
\Delta \omega_{z}<<\Delta \omega_{S} ; \\
{\left[\omega_{z . L} ; \omega_{z . H}\right] \in\left[\omega_{S . L} ; \omega_{S . H}\right] .}
\end{array}\right.
$$

В случае (22) спектр $S_{m}\left(\mathrm{j} \omega_{I F . k}\right)$ полезного сигнала взаимодействует со спектром $S_{z}\left(\mathrm{j} \omega_{I F . k}\right)$ помехи только в пределах полосы $2 \cdot \Delta \omega_{z}$ частот перекрытия их взаимных спектров. В результате двойной корреляционной обработки в пределах полосы $\left(\Delta \omega_{S}-2 \cdot \Delta \omega_{z}\right)$ частот формируется аддитивная смесь суммарного комплексного сигнального $\dot{S}_{m \Sigma}$ и помехового $\dot{S}_{z \Sigma}$ векторов аналогично случаю (13), что определяет соответствующую помеховую составляющую $\sigma_{\tau z}^{2}$ дисперсии погрешности оценки задержки полезного сигнала следующим образом:

$$
\sigma_{\tau z}^{2}=\left(2 P_{z} / P_{S}\right)^{2} .
$$

Сравнительный анализ уравнений (19) и (23) показывает, что при действии узкополосной помехи, перекрывающейся по спектру с полезным сигналом, помехозащищённость существенно зависит от ширины $\Delta \omega_{z}$ спектра помехи и значительно ухудшается по сравнению со случаем, когда помеха не перекрывается по частоте с полезным сигналом.

При действии широкополосной помехи при втором варианте ЭМО начальные условия анализа определяются согласно уравнениям:

$$
\left\{\begin{array}{l}
\Delta \omega_{a}=\Delta \omega_{z} ; \\
\Delta \omega_{S}<<\Delta \omega_{z} ; \\
{\left[\omega_{S . L} ; \omega_{S . H}\right] \in\left[\omega_{z . L} ; \omega_{z . H}\right] .}
\end{array}\right.
$$

Для условий (24) в процессе двойной корреляционной обработки в пределах полосы $\left[\omega_{S . L} ; \omega_{S . H}\right]$ частот спектра полезного сигнала формируется взаимный спектр полезного сигнала и помехи аналогично уравнению (14). Это обусловливает, в свою очередь, эффект полной «пространственной блокировки» полезного сигнала помехой. В результате пеленгование будет осуществляться с аномально большой помеховой составляющей $\sigma_{\tau z}^{2}$ дисперсии погрешности оценки задержки полезного сигнала, аналогично уравнению (19).

При условии $2 \Delta \omega_{S}>\Delta \omega_{z}$ эффект «пространственной блокировки» будет частичным и будет возникать в ограниченной полосе частот, а помехозащищённость будет определяться согласно уравнению:

$$
\sigma_{\tau z}^{2}=\left(\frac{P_{z}}{P_{S}\left(2-\Delta \omega_{z} / \Delta \omega_{S}\right)}\right)^{2} .
$$

Выполним анализ помехозащищённости для третьего варианта ЭМО условий пеленгования, когда спектр $S_{m}\left(\mathrm{j} \omega_{I F . k}\right)$ полезного сигнала и спектр $S_{z}\left(\mathrm{j} \omega_{I F . k}\right)$ помехи перекрываются частично в пределах полосы $\Delta \omega_{S z}$ частот. При действии равнополосной помехи, то есть согласованной по ширине спектра с полезным сигналом, начальные условия анализа следующие:

$$
\left\{\begin{array}{l}
\Delta \omega_{S}=\Delta \omega_{z} ; \\
\Delta \omega_{a}<<\Delta \omega_{S}+\Delta \omega_{z} ; \\
{\left[\omega_{z . L} ; \omega_{z . X}\right] \in\left[\omega_{S . L} ; \omega_{S . H}\right],}
\end{array}\right.
$$

где $\omega_{z . X}-$ верхняя частота полосы перекрытия спектров полезного сигнала и помехи.

Для условий (26) ЭМО влияние помехи на результаты пеленгования будет существенным за счёт эффекта «пространственной блокировки» помехой полезного сигнала в пределах ширины $\left(\Delta \omega_{S}-\Delta \omega_{S z} / 2\right)$ полосы частот его спектра. В пределах полосы $\Delta \omega_{S z} / 2$ эффект «пространственной блокировки» будет отсутствовать. 
В результате с учётом уравнений (23), (26) для указанных условий исследований помеховая составляющая $\sigma_{\tau z}^{2}$ дисперсии погрешности оценки задержки полезного сигнала равняется:

$$
\sigma_{\tau z}^{2}=\frac{P_{z}^{2} \cdot \Delta \omega_{S}^{2}}{P_{S}^{2} \cdot \Delta \omega_{S z}^{2}}
$$

В случае действия узкополосной помехи, спектр которой частично перекрывается со спектром полезного сигнала, условия анализа следующие:

$$
\left\{\begin{array}{l}
\Delta \omega_{S}>>\Delta \omega_{z} ; \\
\Delta \omega_{a}<\Delta \omega_{S}+\Delta \omega_{z} ; \\
{\left[\omega_{z . L} ; \omega_{z . X}\right] \in\left[\omega_{S . L} ; \omega_{S . H}\right] .}
\end{array}\right.
$$

Для условий (28) ЭМО влияние помехи по сравнению со случаем (26) будет меньшим, а эффект «пространственной блокировки» будет присутствовать только в полосе $\left(\Delta \omega_{a}-\Delta \omega_{S}\right)$. В результате с учётом уравнений (23), (27) и (28) для указанных условий исследований помеховая составляющая $\sigma_{\tau z}^{2}$ дисперсии погрешности оценки задержки полезного сигнала равняется:

$$
\sigma_{\tau z}^{2}=\left(\frac{P_{z} \cdot\left(2 \Delta \omega_{z} \cdot \Delta \omega_{S}\right)}{P_{S} \cdot\left(\Delta \omega_{S}-\Delta \omega_{z}+\Delta \omega_{S z}\right)}\right)^{2} .
$$

Анализ уравнения (29) показывает, что помехозащищённость пеленгования с двойной корреляционной обработкой увеличивается с увеличением полосы $\Delta \omega_{S z}$ перекрытия при прочих равных условиях.

В случае действия широкополосной помехи, спектр которой частично перекрывается со спектром полезного сигнала, условия анализа будут следующими:

$$
\left\{\begin{array}{l}
\Delta \omega_{z}>>\Delta \omega_{S} ; \\
\Delta \omega_{a}<\Delta \omega_{S}+\Delta \omega_{z} ; \\
{\left[\omega_{z . L} ; \omega_{z . X}\right] \in\left[\omega_{S . L} ; \omega_{S . H}\right] .}
\end{array} .\right.
$$

Для условий (30) ЭМО влияние помехи будет значительным при наличии эффекта полной «пространственной блокировки» полезного сигнала помехой в пределах всей полосы $\Delta \omega_{S}$ частот полезного сигнала. При этом помеховая составляющая $\sigma_{\tau z}^{2}$ дисперсии погрешности оценки задержки полезного сигнала будет определяться уравнением (8). Улучшение помехозащищённости для условий (30) ЭМО целесообразно достичь за счёт использования дополнительных видов предварительной селекции, таких, например, как пространственная и амплитудная $[5,8]$.

Таким образом, анализ помехозащищённости исследуемого беспоискового спектрального корреляционно-интерферометрического радиопеленгатора с двойной корреляционной обработкой радиоизлучений с расширенным спектром для всех основных вариантов ЭМО выполнен.

Проведены исследования помехозащищённости беспоискового спектрального корреляционноинтерферометрического радиопеленгатора с двойной корреляционной обработкой радиоизлучений с расширенным спектром с помощью разработанной программной модели в среде MathCad для следующих начальных условий: варианты ЭМО - с полным и частичным перекрытием спектров полезного сигнала и помехи; тип полезного сигнала и помехи - непрерывные с линейной частотной модуляцией: $S(t)=A \cdot \sin \left(\omega_{0} t+b t^{2}\right)$; ширина спектра помехи , $\Delta f_{z 2}=5 \mathrm{MГц;} \mathrm{ширина} \mathrm{спектра} \mathrm{сигнала}$

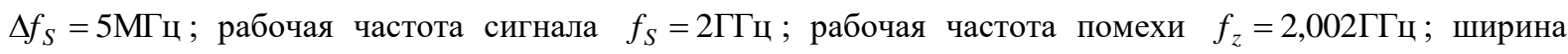
полосы пропускания радиоприёмника $\Delta F_{R}=10 \mathrm{M} ц$; частота дискретизации $f_{d}=20 \mathrm{M \Gamma ц;} \mathrm{заданное}$ направление прихода сигнала $\theta_{S}=80^{\circ}$; заданное направление прихода помехи $\theta_{z}=20^{\circ}$; количество сигнальных отсчётов $N_{S}=16384$; время процесса анализа $T_{a}=N_{S} / f_{d}=16384 / 20 \cdot 10^{6} \Gamma ц=0,8 \mathrm{Mc}$; мощность $P_{n}$ собственного шума намного меньше мощности $P_{z}$ помехи: $P_{n}<<P_{z}$. Полученное семейство зависимостей среднего квадратического отклонения (СКО) $\sigma_{\theta}$ оценки пеленга от отношения $\mathrm{S} / \mathrm{z}$ сигнал / помеха представлено на рисунке 4.

Ряд 1 семейства зависимостей на рисунке 4 соответствует второму варианту ЭМО условий пеленгования при действии узкополосной помехи, спектр которой полностью перекрывается со спектром полезного сигнала в пределах полосы частот 0,5МГц, уравнение (21). Зависимость ряда 1 согласовывается с уравнением (23), соответствуя гиперболическому закону $y=1 / x$ при увеличении мощности $P_{S}$ полезного сигнала. 
Ряд 2 семейства зависимостей на рисунке 4 соответствует третьему варианту ЭМО условий пеленгования при действии равнополосной помехи: $\Delta \omega_{S}=\Delta \omega_{z}$, спектр которой частично перекрывается со спектром полезного сигнала, уравнение (25), в пределах полосы частот $\Delta \omega_{S z} / 2 \pi=3$ МГц . Для данных условий пеленгования характерно существенное влияние эффекта «пространственной блокировки» в пределах полосы частот спектра полезного сигнала шириной $\Delta \omega_{S b} / 2 \pi=2 \mathrm{MГц} \mathrm{.}$

Как показывает сравнительный анализ зависимостей ряда 1 и ряда 2 на рисунке 4 при неизменном отношении сигнал-помеха помехозащищённость исследуемого радиопеленгатора существенно ухудшается при действии помехи, спектр которой частично совмещён со спектром полезного сигнала. Это обусловлено её блокирующим действием и соответствующим эквивалентным уменьшением уровня полезного сигнала на выходе пеленгатора пропорционально ширине $\Delta \omega_{S b}$ полосы частот блокировки, что согласовывается с уравнением (26).

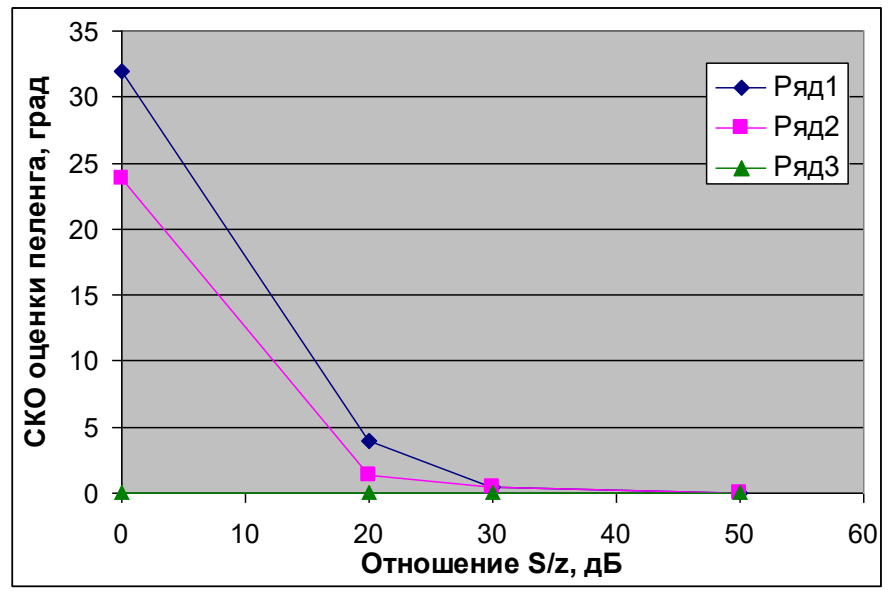

Рис. 4. Семейство зависимостей СКО $\sigma_{\theta}$ оценки пеленга от отношения S/z сигнал / помеха: ряд 1 -

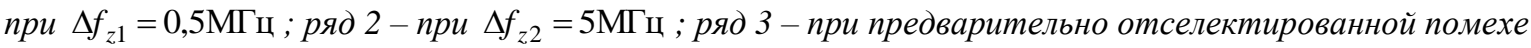

Ряд 3 семейства зависимостей на рисунке 4 соответствует начальным условиям ряда 1, но при использовании пространственной предварительной селекции помехи $[5,8,12]$. В результате помехозащищённость исследуемого пеленгатора существенно улучшается и определяется действием только собственных шумов пеленгационных каналов. При этом всё же остаются определённые энергетические потери полезного сигнала и соответствующее ухудшение точности пеленгования, пропорциональные двойной ширине $2 \Delta \omega_{z}$ полосы частот спектра помехи. Это подтверждает перспективность применения предварительной пространственной селекции помех при реализации беспоискового метода пеленгования с двойной корреляционной обработкой радиоизлучений.

В целом результаты моделирования хорошо согласовываются с результатами теоретического анализа.

Выводы и перспективы будущих исследований. Исследование помехозащищённости беспоискового спектрального корреляционно-интерферометрического радиопеленгатора с двойной корреляционной обработкой радиоизлучений с расширенным спектром показало, что внешние аддитивные помехи имеют значительно большее негативное влияние на точность пеленгования, чем действие собственных шумов пеленгатора при их равных мощностях. Это обусловлено наличием эффекта накопления во времени энергии аддитивной помехи.

Наиболее опасными вариантами ЭМО, которые вызывают появление эффекта «пространственной блокировки» и аномально большую погрешность пеленгования, являются приём равнополосной помехи при отсутствии перекрытия спектров сигнала и помехи, а также при полном перекрытии спектра сигнала и широкополосной помехи. Для других вариантов ЭМО помехозащищённость пеленгования существенно зависит от соотношения ширины спектров полезного сигнала и помехи и отношения сигнал-помеха.

Исследования показали, что основным возможным направлением улучшения помехозащищённости исследуемого пеленгатора является увеличение отношения сигнал-помеха за счёт дополнительной амплитудной, пространственной или поляризационной селекции излучений помехи на этапе разнесённого приёма направленными антенными решётками или на этапе анализа взаимного комплексного спектра при использовании ненаправленных антенн. 
Полученные результаты целесообразно использовать при реализации аппаратуры систем радиомониторинга и радионавигации, функционирующих в сложной ЭМО.

Данные исследования являются продолжением работы по разработке беспоисковых спектральных методов корреляционно-интерферометрического пеленгования.

В дальнейшем необходимо выполнить оценку точности беспоискового спектрального корреляционно-интерферометрического радиопеленгатора для широкополосных сигналов.

\section{Список использованной литературы:}

1. Рембовский А.М. Радиомониторинг - задачи, методы, средства / А.М. Рембовский, А.В. Ашихмин, В.А. Козьмин ; под ред. А.М. Рембовского. - 2-е изд., перераб. и доп. - М. : Горячая линия - Телеком, 2010. $-624 \mathrm{c}$

2. Optimized algorithm for solving phase interferometer ambiguity / S. Van Doan, J.Vesely, P.Janu et al. // 17th International Radar Symposium IRS-2016, Poland : proceedings. - Krakow, 2016. - P. 1-6. DOI: 10.1109/IRS.2016.7497353.

3. Jung-Hoon L. Interferometer direction-finding system with improved DF accuracy using two different array configurations / L.Jung-Hoon, W.Jong-Myung // IEEE Antennas and Wireless Propagation Letters. - 2014. Vol. 14. - P. 719-722. DOI: 10.1109/LAWP.2014.2377291.

4. Ципоренко B.B. Безпошуковий цифровий метод спектрального кореляційно-інтерферометричного радіопеленгування 3 подвійним кореляційним обробленням / B.B. Ципоренко // Всеукраїнський міжвідомчий науково-технічний збірник «Радіотехніка». - 2011. - № 167. - С. 73-77.

5. Long baseline direction finding and localization algorithms for noise radiation source / Y.Jian, C.Wangjie, L.Lei, N.Xiaokang // Signal Processing : 12th International Conference ICSP-2014, Hangzhou, 19-23 October : proceedings. - Mengqi Zhou, 2014. - P. 52-57. DOI: 10.1109/ICOSP.2014.7014968.

6. Ципоренко B.B. Оцінка часової ефективності радіопеленгатора, що працює на основі безпошукового спектрального методу дисперсійно-кореляційного радіопеленгування / В.В. Ципоренко, В.Г. Ципоренко, O.B. Андреєв // Технічна інженерія. - Житомир : Державний університет «Житомирська політехніка». 2020. - № 1 (85). - C. 184-190.

7. Tsyporenko V.V. Analysis of interference immunity of the searchless method of correlation-interferometric direction finding with recostruction of the spatial analytical signal / V.V. Tsyporenko // Eastern-European Journal of Enterprise Technologies. Information and controlling system. - 2017. - Vol. 2, № 9 (86). - P. 45-52. DOI: 10.15587/1729- 4061.2017.96653.

8. Смирнов Ю.А. Радиотехническая разведка / Ю.А. Смирнов. - М. : Воениздат, 2001. - 456 с.

9. Blind spectra separation and direction finding for cognitive radio using temporal correlation-domain ESPRIT / F.Xiao, N.D. Sidiropoulos, M.Wing-Kin, J.Tranter // Acoustics, Speech and Signal Processing : 2014 IEEE International Conference ICASSP, Florence, 4-9 May : proceedings. - Pisa : Marco Luise, 2014. - P. 7749-7753. DOI: 10.1109/ICASSP.2014.6855108.

10. Yingbing W. Studies on circular array interferometer direction finding / W.Yingbing, N.Lirong // 2015 IEEE 6th International Symposium on Microwave, Antenna, Propagation, and EMC Technologies (MAPE), China : proceedings. - Shanghai, 2015. - P. 49-52. DOI: 10.1109/MAPE.2015.7510262.

11. Ципоренко В.B. Дослідження завадостійкості безпошукового цифрового спектрального дисперсійнокореляційного радіопеленгатора для великої антенної бази / В.В. Ципоренко, В.Г. Ципоренко // Вісник Житомирського державного технологічного університету. Серія : Технічні науки. - Житомир : ЖДТУ, 2012. - № 4 (63). - С. 135-143.

12. Слободянюк П.В. Довідник з радіомоніторингу / П.В. Слободянюк, В.Г. Благодарний, В.С. Ступак ; під заг. ред. П.В. Слободянюка. - Ніжин : ТОВ «Видавництво «Аспект-Поліграф», 2008. - 588 с.

13. Proakis J.G. Digital Signal Processing, Principles, Algorithms, and Applications / J.G. Proakis, D.G. Manolakis ; 4th edition - New Jersey : Prentice-Hall, Inc. Upper Saddle River, 2006. - 1077 p.

\section{References:}

1. Rembovskii, A.M., Ashikhmin, A.V. and Koz'min, V.A. (2010), Radiomonitoring - zadachi, metody, sredstva, in Rembovskii, A.M. (ed.), 2nd ed., pererab. i dop., Goryachaya liniya - Telekom, M., 624 p.

2. Van Doan, S., Vesely, J., Janu, P. et al. (2016), «Optimized algorithm for solving phase interferometer ambiguity», 17th International Radar Symposium IRS-2016, Poland, proceedings, Krakow, pp. 1-6, doi: 10.1109/IRS.2016.7497353.

3. Jung-Hoon, L. and Jong-Myung, W. (2014), «Interferometer direction-finding system with improved DF accuracy using two different array configurations», IEEE Antennas and Wireless Propagation Letters, Vol. 14, pp. 719-722, doi: 10.1109/LAWP.2014.2377291.

4. Cyporenko, V.V. (2011), «Bezposhukovyj cyfrovyj metod spektral'nogo koreljacijno-interferometrychnogo radiopelenguvannja z podvijnym koreljacijnym obroblennjam», Vseukrai'ns'kyj mizhvidomchyj naukovo-tehnichnyj zbirnyk «Radiotehnika», No. 167, pp. 73-77.

5. Jian, Y., Wangjie, C., Lei, L. and Xiaokang, N. (2014), «Long baseline direction finding and localization algorithms for noise radiation source», Signal Processing, 12th International Conference ICSP-2014, Hangzhou, 19-23 October, proceedings, Mengqi Zhou, pp. 52-57, doi: 10.1109/ICOSP.2014.7014968. 
6. Cyporenko, V.V., Cyporenko, V.G. and Andrejev, O.V. (2020), «Ocinka chasovoi' efektyvnosti radiopelengatora, shho pracjuje na osnovi bezposhukovogo spektral'nogo metodu dyspersijno-koreljacijnogo radiopelenguvannja», Tehnichna inzhenerija, Derzhavnyj universytet «Zhytomyrs'ka politehnika», Zhytomyr, No. 1 (85), pp. 184-190.

7. Tsyporenko, V.V. (2017), «Analysis of interference immunity of the searchless method of correlationinterferometric direction finding with recostruction of the spatial analytical signal», Eastern-European Journal of Enterprise Technologies. Information and controlling system, Vol. 2, No. 9 (86), pp. 45-52, doi: 10.15587/17294061.2017.96653.

8. Smirnov, Yu.A. (2001), Radiotekhnicheskaya razvedka, Voenizdat, M., 456 p.

9. Xiao, F., Sidiropoulos, N.D., Wing-Kin, M. and Tranter, J. (2014), «Blind spectra separation and direction finding for cognitive radio using temporal correlation-domain ESPRIT», Acoustics, Speech and Signal Processing, 2014 IEEE International Conference ICASSP, Florence, 4-9 May, proceedings, Marco Luise, Pisa, pp. 7749-7753, doi: 10.1109/ICASSP.2014.6855108.

10. Yingbing, W. and Lirong, N. (2015), «Studies on circular array interferometer direction finding», 2015 IEEE 6th International Symposium on Microwave, Antenna, Propagation, and EMC Technologies (MAPE), China, proceedings, Shanghai, pp. 49-52, doi: 10.1109/MAPE.2015.751026.

11. Cyporenko, V.V. and Cyporenko, V.G. (2012), «Doslidzhennja zavadostijkosti bezposhukovogo cyfrovogo spektral'nogo dyspersijno-koreljacijnogo radiopelengatora dlja velykoi' antennoi' bazy», Visnyk Zhytomyrs'kogo derzhavnogo tehnologichnogo universytetu, Serija Tehnichni nauky, ZhDTU, Zhytomyr, No. 4 (63), pp. 135-143.

12. Slobodjanjuk, P.V., Blagodarnyj, V.G. and Stupak, V.S. (2008), Dovidnyk $z$ radiomonitoryngu, in Slobodjanjuk P.V. (ed.), TOV «Vydavnyctvo «Aspekt-Poligraf», Nizhyn, 588 p.

13. Proakis, J.G. and Manolakis, D.G. (2006), Digital Signal Processing, Principles, Algorithms, and Applications, 4th ed., Prentice-Hall, Inc. Upper Saddle River, New Jersey, 1077 p.

Ципоренко Віталій Валентинович - кандидат технічних наук, доцент, доцент кафедри біомедичної інженерії та телекомунікацій Державного університету «Житомирська політехніка».

Наукові інтереси:

- радіомоніторинг;

- цифрова обробка сигналів.

http://orcid.org/0000-0001-8559-006X.

E-mail: tsyporenko.1985@gmail.com.

Ципоренко Валентин Григорович - кандидат технічних наук, доцент, доцент кафедри біомедичної інженерії та телекомунікацій Державного університету «Житомирська політехніка».

Наукові інтереси:

- радіомоніторинг;

- цифрова обробка сигналів.

http://orcid.org/0000-0002-6843-8960.

E-mail: tsyporenkovg@gmail.com.

Стаття надійшла до редакції 19.08.2020. 